\title{
Integrated Shield Edge Diffraction Model for Narrow Obstructing Objects
}

\author{
Tim W. C. Brown, Member, IEEE, Mohsen Khalily, Senior Member, IEEE
}

\begin{abstract}
A new simple and accurate model defined as shield edge diffraction is derived and validated suitable for frequencies above $10 \mathrm{GHz}$ diffracting around obstructions that are narrow compared to the Fresnel zone width. The model includes new simple Fresnel diffraction parameters similar to those used with traditional knife edge diffraction, which can in the same way be integrated into deterministic and empirical path loss models. Capability of the model extends beyond current single and double knife edge models whereby it includes the effects of the antennas' far field distances as well as their gain and phase patterns, which subsequently have a severe effect on the diffraction loss in short range links. The models are validated using both anechoic chamber as well as real environment based measurements at $10-12 \mathrm{GHz}$ and $26 \mathrm{GHz}$.
\end{abstract}

Index Terms-Diffraction, shield edge, knife edge.

\section{INTRODUCTION}

$\mathrm{D}$ FFRACTION has been a vital component in modeling the propagation loss of fixed links for many decades [1]. It is a useful deterministic modeling technique that can accurately determine the additional path loss caused by obstruction to the line of sight. The knife edge diffraction model has been useful for this purpose in that is simple yet accurate. Traditionally, knife edge diffraction is modeled for long range propagation typically at frequencies below $1 \mathrm{GHz}$. The model accounts for major obstructions (e.g. hilly terrain, large buildings or landmarks) that result in significant propagation loss over a wide area in applications like broadcasting and large macro cells for mobile communications. For accurate ray tracing tools, including the diffraction effects is essential. More recently, interest has grown in shorter range links for radar and communications at higher frequencies above $10 \mathrm{GHz}$ [2] where the diffraction scenarios are somewhat different. These instances would include diffraction off the edge of buildings but also a number of objects that are tall in height but relatively narrow compared to the Fresnel zone (e.g. street furniture, tree trunks or information signs), which will result in substantially different diffraction that cannot be modeled by a single knife edge. These diffracting objects will show significant diffraction off both edges and as such recent work in the literature has attempted to address this problem through

T. W. C. Brown and M. Khalily are with the Institute for Communication Systems (home of the 5G Innovation Centre), University of Surrey, Guildford, Surrey, GU2 7XH, UK. (e-mail: tim@brown76.name, m.khalily@surrey.ac.uk). double knife edge diffraction modeling [3]. Such models will take two separate knife edges either side of the diffracting object and combine the diffracted signals together in order to resolve the overall diffraction loss. Other work reported in [4][5] has extended such models to consider diffraction off all four sides of a small diffracting object, while also it has incorporated the effects of the directive transmit and receive antenna patterns. A point of clarity should be noted in this paper whereby double knife edge diffraction can have other meanings such as two consecutive knife edges [6]-[8]. Therefore from this point onwards double knife edge diffraction will be considered as diffraction off two edges of a tall narrow object. Furthermore this paper is only interested in cases where the diffracting object is directly obstructing the line of sight where there are no other multipath effects, as used in geometric theory of diffraction modeling [9].

The motivation for deriving a new model in this paper, which is named shield edge diffraction, overcomes inaccuracies in double knife edge diffraction by integrating the effect of the antennas' far field, directive phase and gain patterns at short range. The paper provides a detailed analysis and validation from measurements to show that this model is suitable for frequencies above $10 \mathrm{GHz}$, where such diffraction scenarios typically occur. The novel contributions in this paper are firstly a model that has newly defined input parameters, secondly a rigorous analysis through simulation and measurement shows the impact of the antennas' effects. Finally a suitable approximation model that incorporates these effects is derived.

Section II will derive and explain the shield edge diffraction model while integrating the antenna effects denoted as far field offset, gain and phase pattern. Section III will validate new simplified models backed by measurement; section IV will demonstrate the application using real outdoor and indoor propagation measurements and finally section $\mathrm{V}$ will end with a conclusion. An appendix with derivations is also provided.

\section{SHIELD EdGE DIFFRACTION}

It is necessary in this section to create simple parameters and derive equations for shield edge diffraction in a similar way to traditional knife edge diffraction. The well known knife edge Fresnel diffraction parameter, $v$ [9] is defined as:

$$
v=\Delta h \sqrt{\frac{2\left(d_{1}+d_{2}\right)}{\lambda d_{1} d_{2}}}
$$


where $\Delta h$ is the displacement from the line of sight to the tip of the knife edge, $\lambda$ is the free space wavelength, while $d_{1}$ and $d_{2}$ are the distances to the diffracting object from the transmitter and receiver respectively. Knife edge diffraction is based upon an infinitely wide diffracting object (or an object substantially wider than the width of the first Fresnel zone) cutting into the edge of the first Fresnel zone. A shield edge defined in this paper is the case where the diffracting object is narrow and is either comparable to or less than the width of the first Fresnel zone. Two new diffraction parameters are defined in this paper: $u$, which corresponds to the width of the shield edge in the center of the Fresnel zone and $w$, which corresponds to the offset of the center the shield edge relative to the line of sight. Shield edge diffraction is illustrated in Fig. 1 (a) showing how $u$ is varied within the Fresnel zone with $w$ fixed at zero, while Fig. 1 (b) shows how $w$ varies within the Fresnel zone with a fixed value of $u$. The length or height in the vertical direction is assumed to be substantially greater than the width within the scope of this work and so only diffraction around the two sides is considered. The transmitter and receiver are labelled as $\mathrm{Tx}$ and $\mathrm{Rx}$ respectively.
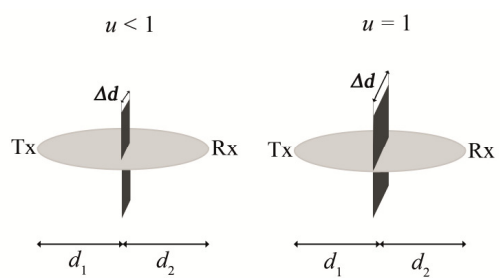

(a)
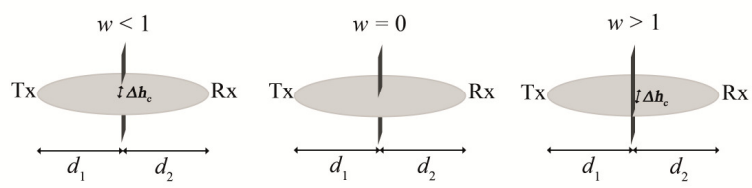

(b)

Fig. 1. Illustration of shield edge diffraction parameters (a) $u$ and (b) $w$.

In calculating diffraction loss, $v$ was used to determine the number of remaining wavelets above the knife edge based on Huygen's principle [10]. For a shield edge, $u$ is defined as follows to determine the remaining wavelets at both edges when the diffracting object is centered at zero:

$$
u=\frac{\Delta d}{2} \sqrt{\frac{2\left(d_{1}+d_{2}\right)}{\lambda d_{1} d_{2}}}
$$

where $\Delta d$ is the width of the diffracting object as shown in Fig. 1 (a). Similar to $v$, the parameter $w$ is defined as follows, though the displacement $\Delta h$ is replaced with $\Delta h_{\mathrm{c}}$ as the offset of the center of the diffracting object from the line of sight as illustrated in Fig. 1 (b):

$$
w=\Delta h_{\mathrm{c}} \sqrt{\frac{2\left(d_{1}+d_{2}\right)}{\lambda d_{1} d_{2}}}
$$

In justifying the basis for defining $u$ and $w$ this way, it is useful to note their function in relation to the Fresnel radius, $r_{\mathrm{F}}$ [9] used to ascertain a zone within which there is significant diffraction if a knife edge intersects it:

$$
r_{\mathrm{F}}=\sqrt{\frac{\lambda d_{1} d_{2}}{d_{1}+d_{2}}}
$$

In knife edge diffraction, when $v=-1, \Delta h$ is equal to $-r_{\mathrm{F}} /$ $\sqrt{2}$, at which point the diffraction loss no longer "oscillates" around $0 \mathrm{~dB}$ [9 Fig. 3.15] but begins to increase with $v$. This same condition is met when $u+w=-1$. When $v=0$, half the wavelets are removed by the knife edge, which is just "kissing" the line of sight, resulting in a $6 \mathrm{~dB}$ loss. The top of the shield edge is also kissing the line of sight when $u+w=0$, while when $-u+w=0$, the bottom of the shield edge is kissing the line of sight. In both these cases however, the loss will be less than or equal to $6 \mathrm{~dB}$ depending on the value of $u$. Finally it is worth noting the case where $v=1$, the point at which $\Delta h$ is equal to $r_{\mathrm{F}} / \sqrt{2}$ and the knife edge diffraction loss is approximated as $1 / \sqrt{2} \pi v$ when $v>1$ [9]. A similar approximation is derived in this paper for cases when $u>1$.

By adapting the work detailed in [10 eq. (13-70)] to form two knife edges, the double knife edge diffraction loss, $L(u, w)$, can be alternatively represented as a shield edge using $u$ and $w$ as the input parameters as follows:

$$
L(u, w)=\frac{E_{\mathrm{diff}}}{E_{\mathrm{s}}}=\frac{1 \pm \mathrm{j}}{2}\left[\int_{u+w}^{\infty} e^{\mp \mathrm{j} \frac{\pi t^{2}}{2}} d t+\int_{-\infty}^{-u+w} e^{\mp \mathrm{j} \frac{\pi t^{2}}{2}} d t\right]
$$

where $E_{\text {diff }}$ is the diffracted field and $E_{\mathrm{s}}$ is the source field. This equation therefore determines the wavelets that are removed due to the presence of the shield edge. As with knife edge diffraction, it is assumed there is no penetration through the diffracting object. Note here that $t$ is not a unit of time, but rather it is used to determine the position of the wavelet such that the exponent term, $\pi t^{2} / 2$, is equal to a phase shift. This phase shift is due to the path difference taking the path from the transmitter, to the point of the wavelet to the receiver relative to the line of sight (i.e. $d_{1}+d_{2}$ ). The proof is shown in the Appendix. The $(1 \pm \mathrm{j}) / 2$ term may be either polarity, but the two exponent terms in equation (5) must be opposite polarity.

The double knife edge diffraction model used in equation (5) requires modification for cases of short propagation distances occurring at high frequencies to include three antenna effects illustrated in Fig. 2: a) The far field offset from each antenna that defines the point where transverse electromagnetic waves predominate to form the Fresnel zone. This offset is calculated by the antenna's Rayleigh distance [11]; b) the phase pattern of the antennas, which cause a phase change to the wavelets and c) the antenna gain patterns, which will cause attenuation to the wavelets. For long propagation distances, these three effects would be negligible as first of all, the Rayleigh distance would be small compared to the propagation distance. Secondly, inspection of Fig. 2 shows 
that if $d_{1}$ and $d_{2}$ become large, the wavelets near the line of sight, which are the most critical, would have negligible change in phase and magnitude due to the antenna patterns. Such effect pre-dominates if either the transmitter, receiver or both are close to the diffracting object. Typically, these effects occur at high frequencies above $10 \mathrm{GHz}$, which propagate at short range and require directive antennas while smaller wavelength increases the Rayleigh distance.

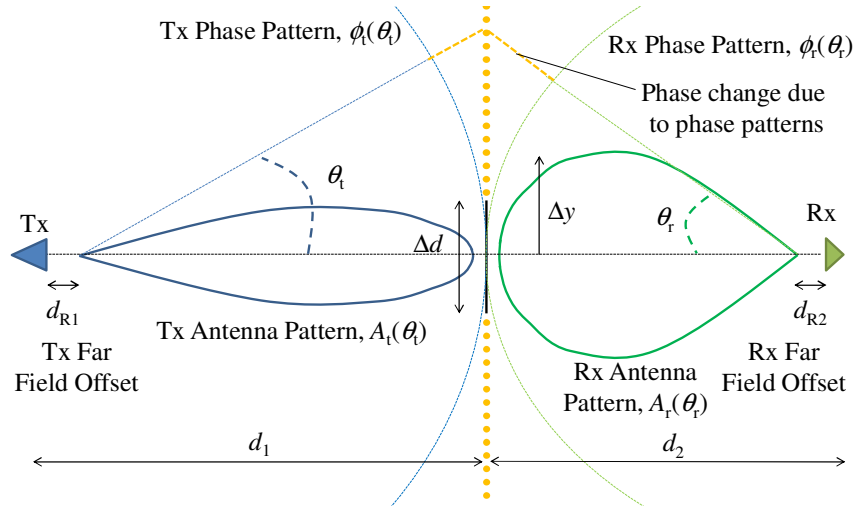

Fig. 2. Illustration of the imposition of a phase error and antenna pattern on the shield edge.

Fig. 2 shows a limited number of diffraction wavelets starting from both the top and bottom of the shield edge, though in reality these wavelets will be infinite in quantity. Two different antenna and phase patterns for the Tx and Rx are shown deliberately, which could in reality be different. At the $\mathrm{Tx}$, each wavelet will be attenuated and phase shifted based on the angular antenna field radiating to it, while the weaker power density subsequently radiated by each wavelet will be further attenuated and phase shifted at the Rx. These angular phase patterns correspond to $\phi_{\mathrm{t}}\left(\theta_{\mathrm{t}}\right)$ and $\phi_{\mathrm{r}}\left(\theta_{\mathrm{r}}\right)$ with gain patterns leading to amplitude patterns $A_{\mathrm{t}}\left(\theta_{\mathrm{t}}\right)$ and $A_{\mathrm{r}}\left(\theta_{\mathrm{r}}\right)$, which are real and the square root of the gain. All patterns can be derived as a function of $t$ since by inspection of Fig. 2 it can be seen that $\tan \theta_{\mathrm{t}}=\Delta y / d_{1}$ and $\tan \theta_{\mathrm{r}}=\Delta y / d_{2}$ while $t$ is related to $\Delta y$ by adapting equation (3) as follows:

$$
\Delta y=t \sqrt{\frac{\lambda d_{1} d_{2}}{2\left(d_{1}+d_{2}\right)}}
$$

The far field offset is due to the Rayleigh distances at both transmit and receive ends, $d_{\mathrm{R} 1}$ and $d_{\mathrm{R} 2}$ shown in Fig. 2. This means that if the diffraction loss is calculated with these distances subtracted from $d_{1}$ and $d_{2}$ then $u$ is effectively increased by offset $u_{\mathrm{R}}$ while $w$ is increased by $w_{\mathrm{R}}$ derived from equations (2) and (3) as follows:

$$
\begin{gathered}
u_{\mathrm{R}}=\frac{\Delta d}{2} \sqrt{\frac{2\left(d_{1}-d_{\mathrm{R} 1}+d_{2}-d_{\mathrm{R} 2}\right)}{\lambda\left(d_{1}-d_{\mathrm{R} 1}\right)\left(d_{2}-d_{\mathrm{R} 2}\right)}}-u \\
w_{\mathrm{R}}=\Delta h_{\mathrm{c}} \sqrt{\frac{2\left(d_{1}-d_{\mathrm{R} 1}+d_{2}-d_{\mathrm{R} 2}\right)}{\lambda\left(d_{1}-d_{\mathrm{R} 1}\right)\left(d_{2}-d_{\mathrm{R} 2}\right)}}-w
\end{gathered}
$$

By incorporating far field offset, as well as amplitude and phase patterns as a function of $t$, equation (5) can be modified to form the integrated shield edge diffraction model for narrow obstructing objects as follows:

$$
L(u, w)=\frac{1}{N_{\mathrm{FW}}}\left[\begin{array}{l}
\int_{u+u_{\mathrm{R}}+w+w_{\mathrm{R}}}^{\infty} A_{\mathrm{t}}(t) A_{\mathrm{r}}(t) e^{\mp \mathrm{j}\left(\frac{\pi t^{2}}{2}+\phi_{\mathrm{t}}(t)+\phi_{\mathrm{r}}(t)\right)} d t \\
+\int_{-\infty}^{-u-u_{\mathrm{R}}+w+w_{\mathrm{R}}} A_{\mathrm{t}}(t) A_{\mathrm{r}}(t) e^{\mp j\left(\frac{\pi t^{2}}{2}+\phi_{\mathrm{t}}(t)+\phi_{\mathrm{r}}(t)\right)} d t
\end{array}\right]
$$

where a normalization term defining the full wavelet field magnitude, $N_{\mathrm{FW}}$, has to be introduced as follows:

$$
\left.N_{\mathrm{FW}}=\int_{-\infty}^{\infty} A_{\mathrm{t}}(t) A_{\mathrm{r}}(t) e^{\mp \mathrm{F}\left(\frac{\pi t^{2}}{2}+\phi_{\mathrm{t}}(t)+\phi_{\mathrm{r}}(t)\right.}\right) d t
$$

Note $N_{\mathrm{FW}}$ reduces to $2 /(1 \pm \mathrm{j})$ and equation (9) reduces to equation (5) if $u_{\mathrm{R}}=w_{\mathrm{R}}=\phi_{\mathrm{t}}(t)=\phi_{\mathrm{r}}(t)=0^{\circ}$ and $A_{\mathrm{t}}(t)=A_{\mathrm{r}}(t)=1$. This would of course correspond to zero or negligible far field offset, antenna phase and gain pattern effects.

To quantify the impact of the far field offset, this is analyzed in Fig. 3, where $d_{1}=d_{2}=d$ and $w=0$. Additionally $d_{\mathrm{R} 1}=d_{\mathrm{R} 2}=d_{\mathrm{R}}$ so that the effect of the far field offset can be found through setting the ratio $d_{\mathrm{R}} / d$ between zero and 1 . Clearly for low $d_{\mathrm{R}} / d$ below 0.2 , the far field offset has negligible effect and the diffraction loss is close to double knife edge, while above 0.2 the extra diffraction loss caused is substantial in excess of $3 \mathrm{~dB}$. This effect is likely to occur at higher frequencies, or small wavelengths at short range since $d_{\mathrm{R}}=2 D^{2} / \lambda[11]$, where $D$ is the maximum antenna dimension. Also if $d_{1}>d_{2}$ and $d_{\mathrm{R} 1} / \mathrm{d}_{1}<<0.2$, similar results to that in Fig. 3 would be found if $d_{\mathrm{R} 2} / \mathrm{d}_{2}>0.2$, or in vice versa.

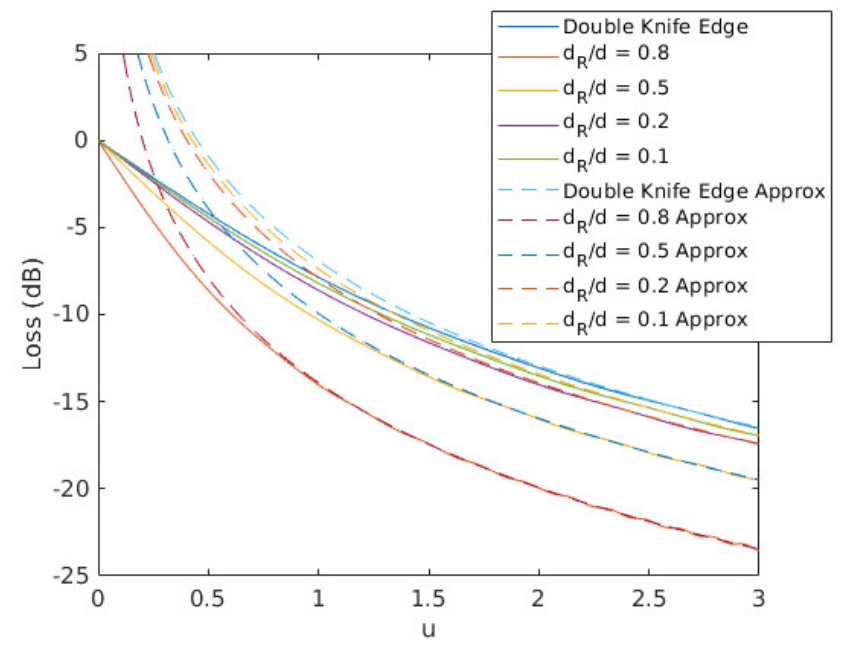

Fig. 3. Plot of the diffraction loss vs $u$ when $w=0$ comparing the impact of far field offset on the wavelets for fixed values of $d_{1}=d_{2}=d, d_{\mathrm{R} 1}=d_{\mathrm{R} 2}=d_{\mathrm{R}}$ with different ratios of $d_{\mathrm{R}} / d$.

Also shown in Fig. 3 are approximation curves that are found to be in agreement with the actual diffraction losses when $u>1$. The approximation can be derived from the well known equation for knife edge diffraction for $v>1$ stated earlier whereby $L(v) \approx 1 / \sqrt{2} \pi v$. This equation is adapted by 
forming two separate knife edges but at the same time accommodating the far field offset $u_{\mathrm{R}}$ from equation (7):

$$
L(u) \approx \frac{1}{\sqrt{2} \pi\left|u+u_{\mathrm{R}}\right|}+\frac{1}{\sqrt{2} \pi\left|-u-u_{\mathrm{R}}\right|}=\frac{\sqrt{2}}{\pi\left(u+u_{\mathrm{R}}\right)}
$$

Clearly equation $(11)$ reduces to $L(u) \approx \sqrt{2} / \pi u$, with no far field offset. Unfortunately, an approximation cannot be derived in a similar way for cases where $w$ is non zero. Thus, approximation is resolved by curves fitting in the next section.

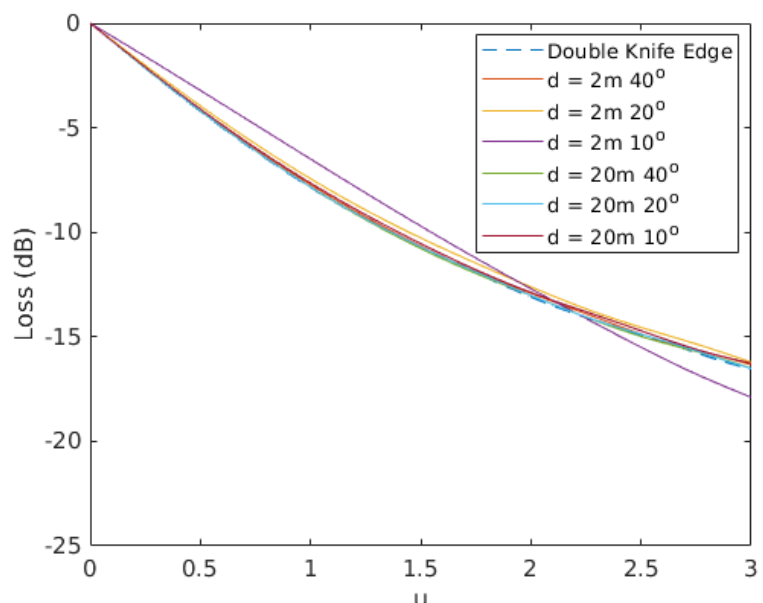

(a)

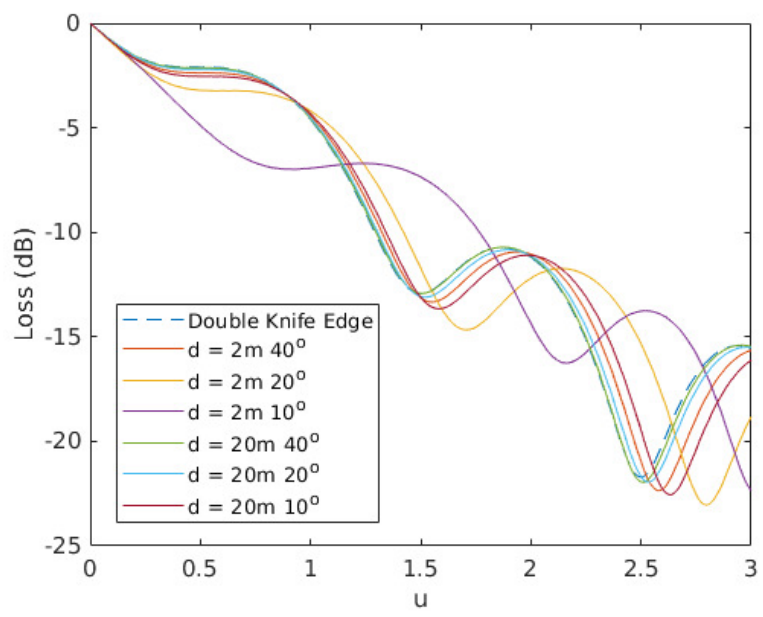

(b)

Fig. 4. Plot of the diffraction loss vs $u$ when (a) $w=0$ and (b) $w=1$ while the distances $d_{1}=d_{2}=d$ for fixed $d$ at a frequency of $10 \mathrm{GHz}$.

The effect of the antenna phase pattern, assuming no far field offset or gain pattern effects, is analyzed in Fig. 4 for two cases where $w=0$ and $w=1$. In order to quantify the impact, it is necessary to not only control the phase pattern itself, but to set a short distance from the diffracting object and an arbitrary frequency. Therefore $10 \mathrm{GHz}$ is chosen with distance $d=d_{1}=d_{2}$ having fixed values of $2 \mathrm{~m}$ and $20 \mathrm{~m}$. The phase pattern of the antennas is defined by a simple function where $\theta_{\mathrm{t}}=\theta_{\mathrm{r}}=\theta$ as follows:

$$
\phi_{\mathrm{t}}\left(\theta_{\mathrm{t}}\right)=\phi_{\mathrm{r}}\left(\theta_{\mathrm{r}}\right)=180(\cos k \theta-1)
$$

The variable $k$ can be used to control the " $90^{\circ}$ beamwidth" (i.e. the beamwidth over which the phase changes by $-90^{\circ}$ with $0^{\circ}$ at boresight) to form narrow and wide patterns. Table I shows values of $k$ and corresponding $90^{\circ}$ beamwidths formed.

\begin{tabular}{|c|c|}
\hline$k$ & $90^{\circ}$ Beamwidth $\left(^{\circ}\right)$ \\
\hline 3 & 40 \\
\hline 6 & 20 \\
\hline 12 & 10 \\
\hline
\end{tabular}

Table I - Values of $k$ for selected $90^{\circ}$ beamwidths used in equation (12).

Analysis of Fig. 4 (a) and (b) shows a negligible effect on the diffraction loss when the $90^{\circ}$ beamwidth is above $10^{\circ}$, though below this beamwidth the effect is more pronounced, especially when $w$ is non zero and only $2 \mathrm{~m}$ from the diffracting object. In many practical cases, the $90^{\circ}$ beamwidth would not get this narrow, though similar characteristics to this are found to occur either where $d_{2} \gg d_{1}$ or $d_{1} \gg d_{2}$ and $d_{2}$ or $d_{1}$ respectively is shorter than $2 \mathrm{~m}$. Phase pattern effects are therefore only of concern where the transmitter or receiver is very close to the diffracting object. A frequency of $10 \mathrm{GHz}$ was chosen in this instance, though if it is increased with $d$ unchanged, the Fresnel zone would be physically narrower resulting in little phase change to the wavelets near to the line of sight and therefore little change to the diffraction loss. Conversely, frequencies lower than $10 \mathrm{GHz}$ with a wider Fresnel zone would see more phase pattern effect though such diffraction scenarios at short range are not common.

The effect of antenna gain patterns only are analyzed in Fig. 5 also for $w=0$ and $w=1$ where $d_{1}=d_{2}=d$ but for clarity $d$ is given fixed values of $10 \mathrm{~m}$ and $100 \mathrm{~m}$ at a frequency of $10 \mathrm{GHz}$. The chosen $3 \mathrm{~dB}$ beamwidths are $5^{\circ}, 10^{\circ}$ and $20^{\circ}$. The following synthetic antenna pattern model was applied and is defined in $\mathrm{dB}$ as follows:

$$
A_{\mathrm{t}}\left(\theta_{\mathrm{t}}\right)(\mathrm{dB})=\max \left[-180,40 \log _{10}\left|\cos \left(k \theta_{\mathrm{t}}\right)\right|\right]
$$

Table II shows values of $k$ and corresponding $3 \mathrm{~dB}$ beamwidths formed. The Rx antenna was assumed to be isotropic.

\begin{tabular}{|c|c|}
\hline$k$ & 3dB Beamwidth $\left(^{\circ}\right)$ \\
\hline 12 & 5 \\
\hline 7 & 10 \\
\hline 3 & 20 \\
\hline
\end{tabular}

Table II - Derived values of $k$ for selected beamwidths used in equation (13).

The results in Fig. 5 show that with a $3 \mathrm{~dB}$ beamwidth less than $10^{\circ}$ and when the distance from the diffracting object of either the transmitter or receiver, or both, is less than $10 \mathrm{~m}$, the antenna pattern will impose attenuation on the wavelets such that the diffraction loss deviates in the order of several $\mathrm{dB}$ when $u>1$. The antenna gain pattern effects are therefore more significant than that of the phase patterns in practical scenarios and modeling their effects becomes more complex when $w$ is non zero as shown in Fig. 5 (b) even for wider $3 \mathrm{~dB}$ beamwidths. This is due to asymmetry in magnitude of the wavelets either side of the shield edge. Similar to Fig. 4 (b), fluctuations are observed due to varying constructive and destructive superimposed fields from both edges. 


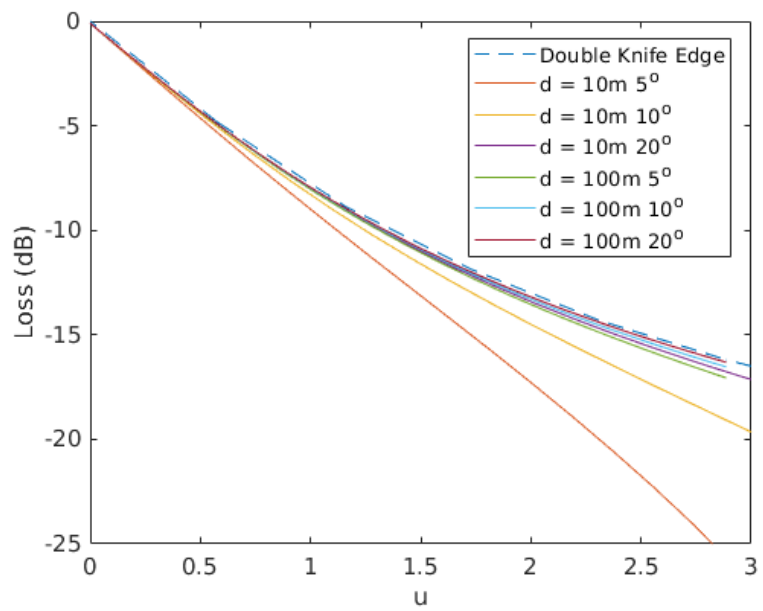

(a)

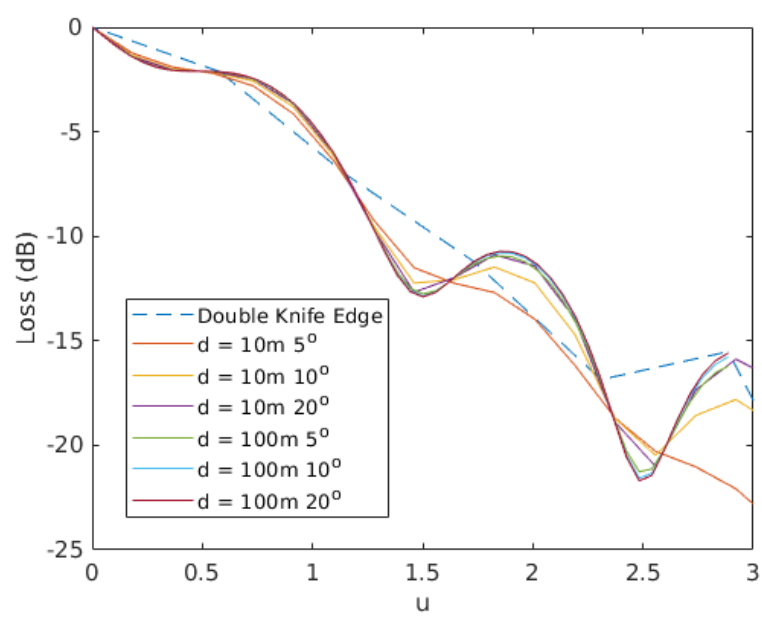

(b)

Fig. 5. Plot of the diffraction loss vs $u$ when (a) $w=0$ and (b) $w=1$ comparing the impact of antenna pattern with varying beamwidths and distance $d_{1}=d_{2}=d$ at a frequency of $10 \mathrm{GHz}$.

It is also worth commenting at this point that results are presented in this section for values of $u$ between 0 and 3 . This is because when $u>3$, the shield edge can be considered to be big enough that it becomes a knife edge; therefore the knife edge diffraction can be calculated from the nearest edge as the other edge would be assumed to have negligible diffraction. To conclude this section it is noteworthy that the knife edge diffraction model in [9 eq (3.26)] can be adapted in the same way to incorporate antenna far field offset, gain and phase pattern effects as follows:

$$
\left.L(v)=\frac{1}{N_{\mathrm{FW}}} \int_{v+\nu_{\mathrm{R}}}^{\infty} A_{\mathrm{t}}(t) A_{r}(t) e^{\mp j\left(\frac{\pi t^{2}}{2}+\phi_{t}(t)+\dot{\phi}_{r}(t)\right.}\right) d t
$$

where:

$$
v_{\mathrm{R}}=\Delta h \sqrt{\frac{2\left(d_{1}-d_{\mathrm{R} 1}+d_{2}-d_{\mathrm{R} 2}\right)}{\lambda\left(d_{1}-d_{\mathrm{R} 1}\right)\left(d_{2}-d_{\mathrm{R} 2}\right)}}-v
$$

\section{Model VALidATION - ANeChoic CHAMBer BASED MEASUREMENTS}

Initial measurements were conducted in free space conditions in an anechoic chamber the range of $10-12 \mathrm{GHz}$ using two identical directional horn antennas facing each other at a distance of $3 \mathrm{~m}$. Shield edge diffracting objects were placed midway, thus $d_{1}=d_{2}=1.5 \mathrm{~m}$. As several frequency points (corresponding to different values of $\lambda$ ) were measured in the frequency range, this enabled several data points with different values of $u$ and $w$ to be generated for three different shield edges made from card and aluminum foil coating, which were $70 \mathrm{~mm}, 140 \mathrm{~mm}$ and $210 \mathrm{~mm}$ wide.

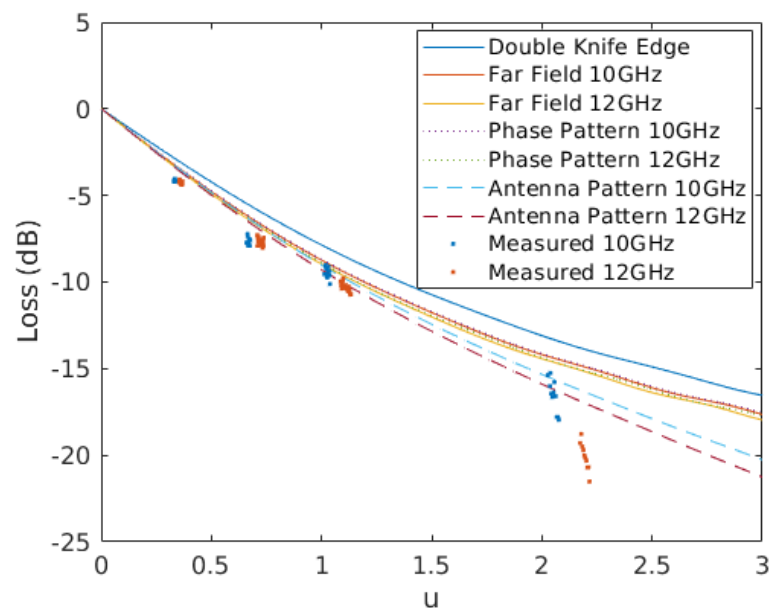

Fig. 6. Validation of the diffraction loss vs $u$ when $w=0$ against measurement data in free space.

The measurement results for the case when $w=0$ are compared against the model in Fig. 6 to identify the effects of far field offset, then the phase pattern and the gain pattern in addition. It should be noted the measured samples at $10 \mathrm{GHz}$ were in the range 10 to $10.5 \mathrm{GHz}$, while the measured samples at $12 \mathrm{GHz}$ were in the range 12 to $12.5 \mathrm{GHz}$. The gain and phase patterns were modeled by integration of the wavelets at the mouth of the horn using well known formulae [12] and validated by pattern measurements. Fig. 6 shows the far field offset in these measurements has the greatest impact while the phase pattern has negligible effect since its $90^{\circ}$ beamwidth is wide, greater than $40^{\circ}$. The antenna patterns had $3 \mathrm{~dB}$ beamwidths of $23^{\circ}$ and $20^{\circ}$ at $10 \mathrm{GHz}$ and $12 \mathrm{GHz}$ respectively causing the loss to be more significant where $u>1.5$.

Where $u<1.5$, measured data and theory are in good agreement with less than $1 \mathrm{~dB}$ difference in Fig. 6. Such error is due to the uncertainty of where the shield edge was positioned as accuracy to the nearest $5 \mathrm{~mm}$ was possible, which corresponds to such magnitude of error. In the case where $u>1.5$, the measurements have a difference of $2-3 \mathrm{~dB}$. In such cases, all of the antenna beamwidth is incident on the shield edge while there was found to be asymmetry in the measured antenna patterns incident on either side of the shield edge. This asymmetry in magnitude of the wavelets caused extra diffraction loss that could not be easily modeled.

Measured data in the same setup is also compared with the model for cases when $w$ is non zero in Fig. 7 vs $u$ with fixed $w$ and Fig. 8 vs $w$ with fixed $u$. For clarity, only $10 \mathrm{GHz}$ 
measured data is used here and the modeled results for just far field offset as well as antenna and phase patterns combined are shown. It can be seen that the measurements and model are in good agreement though it is clear that when $w$ is non zero, the diffraction loss has a fluctuating characteristic as either $u$ or $w$ change. This is explained by diffracted fields from both sides superimposing either add constructively or destructively. This effect becomes less pronounced when $w>2$ and $u<2$ since the shield edge is moving away from the Fresnel zone. The measured data and model has differences up to $6 \mathrm{~dB}$ as such fluctuations cause measurements to be substantially more sensitive to error when $w$ is non zero. Nonetheless, the measured diffraction losses are clearly within the expected range that the fluctuations occur.

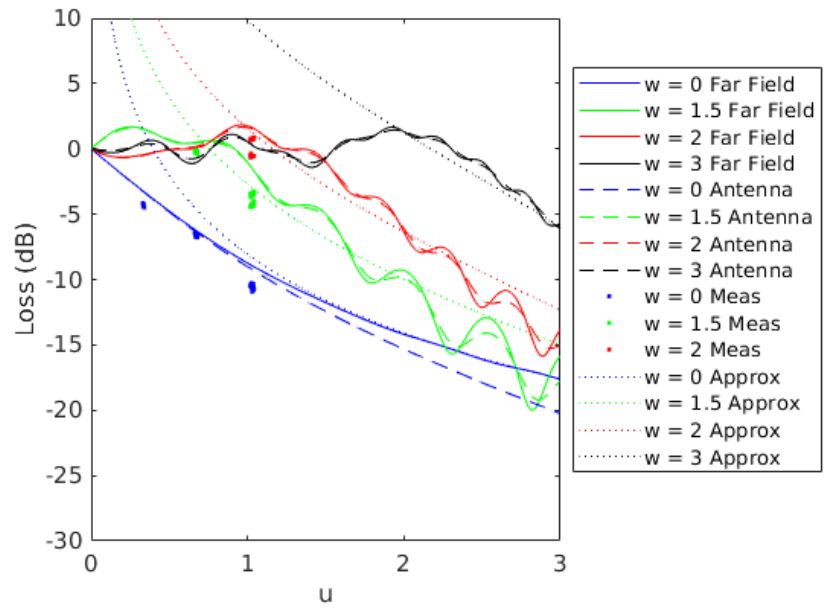

Fig. 7. Validation of the diffraction loss and approximation vs $u$ for fixed values of $w$ against measurement data in free space at $10 \mathrm{GHz}$.

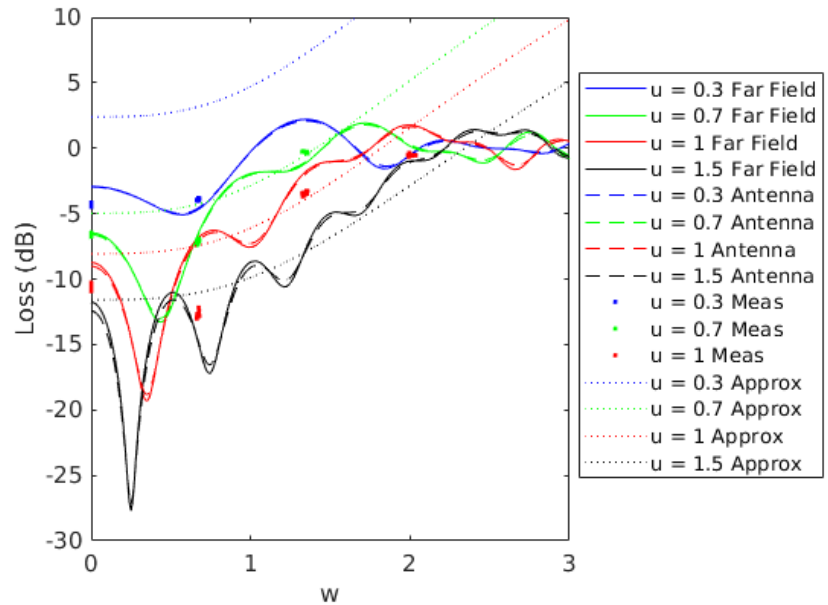

Fig. 8. Validation of the diffraction loss and approximation vs $w$ for fixed values of $u$ against measurement data in free space at $10 \mathrm{GHz}$.

Finally, approximation functions are chosen (where others could be designed by trial and error) suitable for a minimum expected loss and defined in equations (16)-(19) to fit with the actual diffraction loss with far field offset. It is visible from Fig. 7 and Fig. 8 that the approximation is suitable where $u>1$ though it should be noted that in practice, the approximated loss has a maximum value of $0 \mathrm{~dB}$ as defined in equation (16), but this cap is not plotted for clarity. Note that in the case when $w=0$, the approximation simplifies to equation (11).

$$
L(u, w) \approx \min \left(b|w|^{a}+c, 1\right)
$$

where:

$$
\begin{gathered}
a=\frac{u+u_{\mathrm{R}}}{1000}+3 \\
b=\frac{1}{10\left(u+u_{\mathrm{R}}\right)}-\frac{1}{50} \\
c=\frac{\sqrt{2}}{\pi\left(u+u_{\mathrm{R}}\right)}
\end{gathered}
$$

\section{MODEL COMPARISON WITH EXAMPLE REAL ENVIRONMENT DIFFRACTION SCENARIOS}

To demonstrate the application of the model, it is compared with measurements from two real life diffraction scenarios, one indoor and one outdoor, where results show that real objects can be represented as shield edges.

\section{A. Measurement Setup}

The measurement setup has a plan view shown in Fig. 9. A Tx horn with a wide beamwidth of $61^{\circ}$ was used and had a gain of $6.8 \mathrm{dBi}$, while the receiver had a narrower beam of $10^{\circ}$ with a gain of $24 \mathrm{dBi}$. The center frequency was $26 \mathrm{GHz}$ and the distances were set so that $d_{1}=d_{2}=2.5 \mathrm{~m}$. These distances did slightly vary in the calculation as the Rx horn was moved $\pm 1 \mathrm{~m}$ in $0.2 \mathrm{~m}$ steps as shown in Fig. 9 while at each position the antenna was rotated to point directly to the Rx, from which the diffraction could be measured and the theoretical diffraction evaluated.

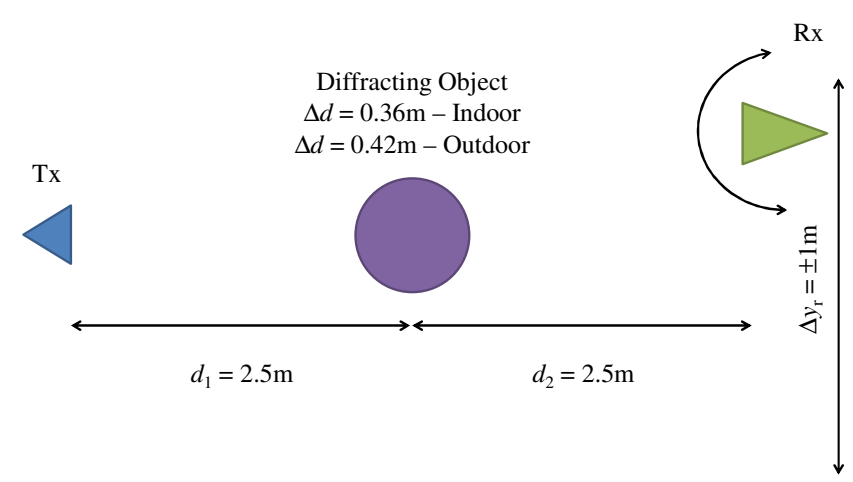

Fig. 9. Illustration of the measurement setup for the outdoor and indoor diffraction measurement where the diffracting object was a pillar and the outdoor object was a tree trunk.

At the Tx, a Rohde \& Schwarz signal generator R\&S®SMW200A was used to transmit continuous wave at $26 \mathrm{GHz}$ with power of $17 \mathrm{dBm}$ and modulated with a sounding waveform, Frank-Zadoff-Chu 65535. The received signal was recorded by an R\&S®FSW67 spectrum analyzer to capture the I/Q data that was sent to a R\&S®RTO1044 where a $2 \mathrm{GHz}$ bandwidth impulse response was recorded and the data was processed by control software. A rotator table was also used to change the angle of the $\mathrm{Rx}$ horn, which is shown in photographs of the two measurement environments in Fig. 10. The indoor diffracting object was a cylindrical building pillar while the outdoor object was a tree trunk, thus a shield edge 
could be seen at any angle. The attenuation of both these objects is sufficient to assume they have no penetration.

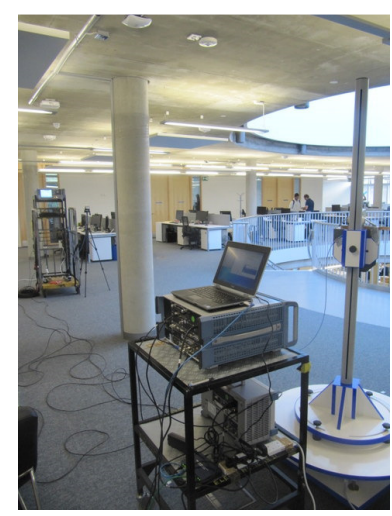

(a)

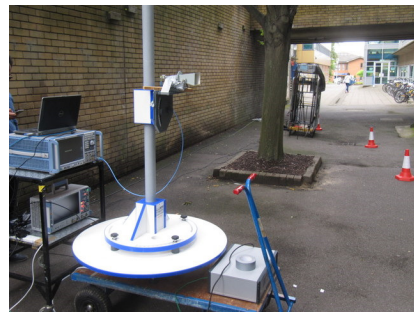

(b)
Fig. 10. Illustration of the measurement setup for the (a) outdoor and (b) indoor diffraction measurement where the diffracting object was a pillar and the outdoor object was a tree trunk.

\section{B. Measurement and Model Comparison}

Measured and modelled results are compared for both the outdoor case in Fig. 11 and indoor case in Fig. 12. The peak impulse response at each point was measured and the free space path loss, antenna gains and cable losses are normalized out in order to make a direct comparison of diffraction loss. As the diffracting object has a fixed width, $u$ is fixed and $w$ is variable as the Rx was moved with varying $\Delta y_{\mathrm{r}}$ of $\pm 1 \mathrm{~m}$. The points of interest here are when $w$ is between -2 and 2. In this range, there is pure shield edge diffraction with no multipath (with the exception of the indoor case when $w=1.5$ ). Clearly it can be seen in this region that measured data is in agreement with results accommodating the far field offset and the antenna gain pattern effects, where the Rx antenna has a large Rayleigh distance of $0.8 \mathrm{~m}$ and a directive pattern. These combined effects cause some $10 \mathrm{~dB}$ extra diffraction loss. Where $w<-2$ and $w>2$ measurements are not in agreement with model because the $0.5 \mathrm{~ns}$ time bin where the peak was measured also captured some reflections off the diffracting object and possibly the nearby walls/furniture. Hence loss in these cases is a combination of diffraction and multipath.

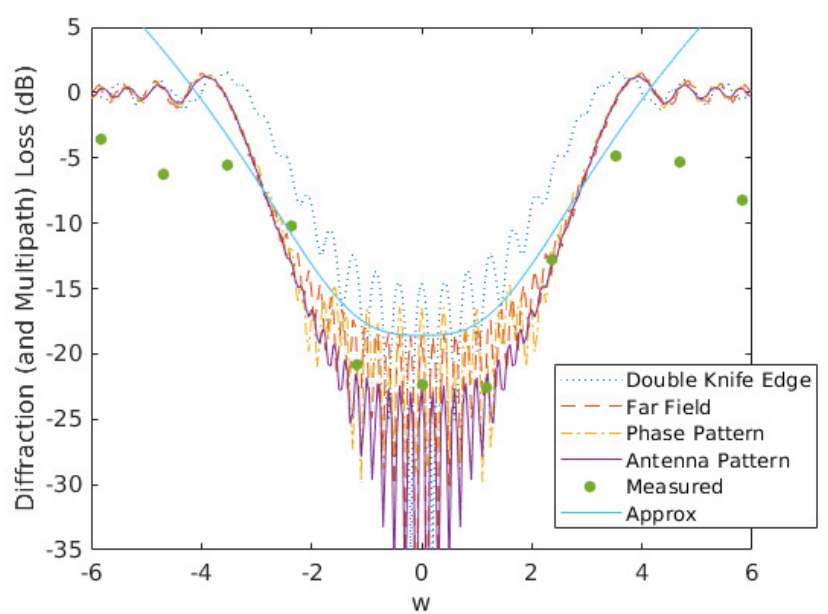

Fig. 11. Model comparison for outdoor case with far field offset, antenna phase and gain pattern effects against the measured and approximated results.
As a final observation to support analysis in section II, the antenna phase pattern makes little difference to the diffraction loss, while the far field offset causes approximately $4 \mathrm{~dB}$ additional loss and the antenna pattern a further $6 \mathrm{~dB}$ loss in the worst case scenario compared with the double knife edge.

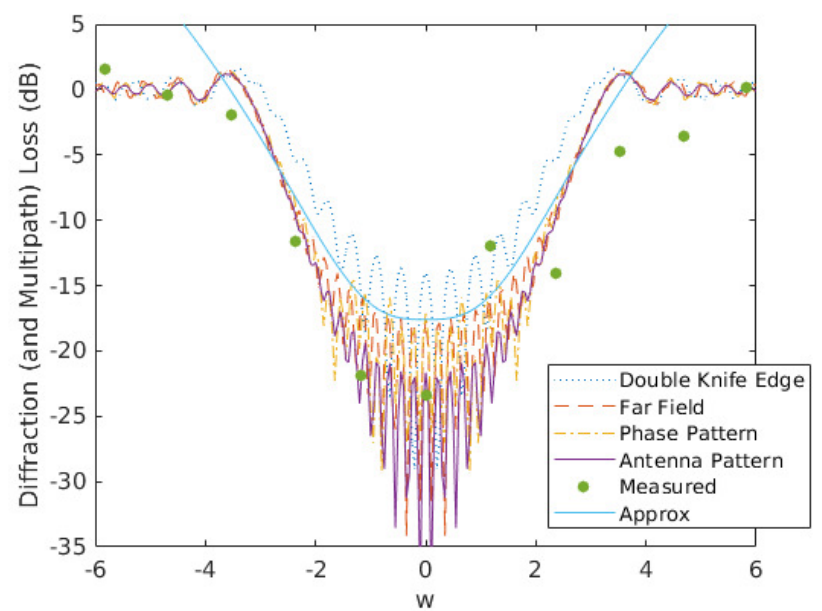

Fig. 12. Model comparison for indoor case with far field offset, antenna phase and gain pattern effects against the measured and approximated results.

\section{CONCLUSION}

An integrated shield edge diffraction model has been derived and validated by measurements. The model extends the double knife edge diffraction model to incorporate offset due to the antennas' far fields, their phase patterns and their directive gain patterns and forms a new approach to reliably approximate the diffraction loss around a shield edge. The model is suitable for frequencies above $10 \mathrm{GHz}$ where there is short propagation range, directive antennas are used and the Rayleigh distance can be significant in magnitude compared to the distance to the diffracting object. Such scenarios at these frequencies require appropriate correction to the calculation or approximation of diffraction loss.

\section{APPENDIX - PROOF OF THE WAVELET PHASOR COMPONENT}

The geometry setup for the shield edge diffraction is illustrated in Fig. A1, with dimensions as in Fig. 2. Additional dimensions are the Fresnel radius, $r_{\mathrm{F}}$, the path distance around the shield edges, $r_{1 \mathrm{a}}+r_{1 \mathrm{~b}}$ and $r_{2 \mathrm{a}}+r_{2 \mathrm{~b}}$ from the transmitter $(\mathrm{Tx})$ to the receiver $(\mathrm{Rx})$.

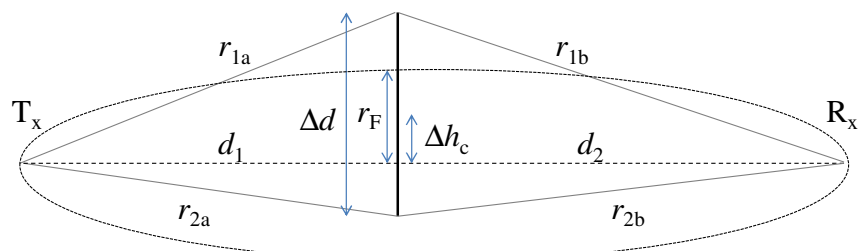

Fig. A1 - Illustration of the path lengths to the top and bottom edges of a shield edge.

The phase shift relative to the line of sight to the top edge, $\phi_{\mathrm{te}}$ can be derived as:

$$
\phi_{\mathrm{te}}=\beta\left(r_{1 \mathrm{a}}+r_{1 \mathrm{~b}}-d_{1}-d_{2}\right)
$$


where $\beta$ is the phase constant equal to $2 \pi / \lambda$. By using Pythagoras' theorem, also factoring out $d_{1}$ and $d_{2}$ :

$r_{\mathrm{la}}+r_{\mathrm{lb}}=d_{1} \sqrt{d_{1}^{2}+\left(\frac{\Delta d / 2+\Delta h_{\mathrm{c}}}{d_{1}}\right)^{2}}+d_{2} \sqrt{1+\left(\frac{\Delta d / 2+\Delta h_{\mathrm{c}}}{d_{2}}\right)^{2}}$

Using the approximation that $\sqrt{1+x} \approx 1+x / 2$ this can be integrated into equation (A2) and subsequently into equation (A1) so that the phase shift simplifies to:

$$
\phi_{\mathrm{te}}=\frac{2 \pi}{4}\left[\left(\frac{\Delta d}{2}+\Delta h_{\mathrm{c}}\right) \sqrt{\frac{2\left(d_{1}+d_{2}\right)}{\lambda d_{1} d_{2}}}\right]^{2}=\frac{\pi(u+w)^{2}}{2}
$$

The phase shift from the bottom of the shield edge relative to the line of sight, $\phi_{\mathrm{be}}$ can be similarly derived as follows:

$$
\phi_{\mathrm{be}}=\frac{2 \pi}{4}\left[\left(-\frac{\Delta d}{2}+\Delta h_{\mathrm{c}}\right) \sqrt{\frac{2\left(d_{1}+d_{2}\right)}{\lambda d_{1} d_{2}}}\right]^{2}=\frac{\pi(-u+w)^{2}}{2}
$$

The term $t$ used in equation (5) starts at either $(u+w)$ and increases to $\infty$, or starts at $(-u+w)$ and decreases to $-\infty$. It can be inferred from equations (A3) and (A4) that for any value of $t$, the phase $\xi$ to any wavelet relative to the line of sight the line of sight is therefore:

$$
\xi=\frac{\pi t^{2}}{2}
$$

\section{ACKNOWLEDGEMENT}

The 5G Innovation Centre, University of Surrey, UK, is acknowledged in forming the motivation for this work.

\section{REFERENCES}

[1] L. E. Vogler, "An attenuation function for multiple knife-edge diffraction", Radio Science, vol. 17, no. 06, pp. 1541-1546, Nov.-Dec. 1982.

[2] M. Flament, M. Unbehaun, "Impact of shadow-fading in a mm-wave band wireless network", International Symposium on Wireless Personal Multimedia Communications, vol. 1, pp.427-432, 2000.

[3] M. Jacob, S. Priebe, R. Dickhoff, T. Kleine-Ostmann, T. Schrader, T. Kürner, "Diffraction in $\mathrm{mm}$ and Sub-mm Wave Indoor Propagation Channels", IEEE Transactions on Microwave Theory and Techniques, vol. 60, no. 3, March 2012, pp833-844.

[4] G. R. MacCartney, S. Deng, S. Sun and T. S. Rappaport, "MillimeterWave Human Blockage at $73 \mathrm{GHz}$ with a Simple Double Knife-Edge Diffraction Model and Extension for Directional Antennas," IEEE Vehicular Technology Conference, 2016, pp. 1-6.

[5] "METIS Channel Models", Mobile and wireless communications Enablers for the Twenty-twenty Information Society (METIS), Deliverable D1.4, ICT-317669-METIS/D1.4, July 2015.

[6] G. Millington, R. Hewitt and F. S. Immirzi, "Double knife-edge diffraction in field-strength predictions", Proceedings of the IEE, Part C: Monographs, vol. 109, no. 16, pp. 419-429, September 1962.

[7] G. L. James and G. T. Poulton, "Double knife-edge diffraction for curved screens," IEE Journal on Microwaves, Optics and Acoustics, vol. 3, no. 6, pp. 221-223, November 1979.

[8] I. Rodríguez-Rodríguez, M-T. Martínez Inglés, J-V. Rodríguez, "On the possibility of estimating the multiple-diffraction losses of a rectangular obstacle at $\mathrm{mm}$-wave frequencies from the corresponding double knife-edge results", AEU International Journal of Electronics and Communications, Vol. 82, 2017, pp516-519.
[9] S. R. Saunders, A. Aragón-Zavala, "Antennas and Propagation for Wireless Communication Systems, 2007, Wiley, UK.

[10] E. C. Jordan, K. G. Balman, "Electromagnetic Waves and Radiating Systems", Second Edition, 1968, Prentice-Hall, UK.

[11] C. A. Balanis, "Antenna Theory, Analysis and design”, Wiley, 2005, USA.

[12] R. E. Collin, "Antennas and Radiowave Propagation", McGraw Hill, 1985 . 